\title{
Interplay of current noise and delayed optical feedback on the dynamics of semiconductor lasers
}

\author{
Miguel C. Soriano, Thomas Berkvens, Guy Van der Sande, Guy Verschaffelt, Jan Danckaert, and Ingo Fischer
}

\begin{abstract}
We study the influence of low frequency current noise on a single-mode semiconductor laser subject to external optical feedback. We experimentally show that the addition of current noise modifies the power spectrum of the chaotic intensity fluctuations of the laser subject to feedback. From the power spectrum measurements, we can deduce interactions among the feedback induced dynamics and the current noise. Numerical modelling based on a Lang-Kobayashi rate-equations provides additional insight as the phase of the electric field can be analyzed. We find that the noise affects the coherence of the chaotic oscillations, thereby being able to alter their characteristics significantly as long as this coherence exists.
\end{abstract}

Index Terms-Semiconductor lasers, optical feedback, dynamics, current noise.

\section{INTRODUCTION}

Semiconductor lasers are known to be very sensitive to external perturbations such as feedback from a distant mirror [1], phase conjugate feedback [2], electro optical feedback [3], injection of light from another laser [4], delayed coupling [5] and current modulation [6]. As a consequence of these perturbations, the output of these lasers may show complex dynamical behavior [7].

Here we concentrate on the influence and the interplay of external current noise and external optical feedback on the dynamical properties of semiconductor lasers, including the relaxation oscillation dynamics and delay induced instabilities. On the one hand, a large amount of current noise can modify the basic dynamical properties of semiconductor lasers [8]. On the other hand, a semiconductor laser subject to optical feedback can exhibit complex dynamics. The strong chaotic fluctuations that delayed optical feedback induces

Part of this work was funded by Ministerio de Ciencia e Innovación (MICINN), Spain, Fondo Europeo de Desarrollo Regional (FEDER) and Consejo Superior Investigaciones Cientificas (Spain) under Projects TEC200914101 (DeCoDicA) and Proyectos Intramurales Especiales [200950I190]. The authors would like to acknowledge financial support of the IAP P6/10 network "Photonics@be". This work was also supported by the Belgian Science Policy Office under grant IAP-VI10. We acknowledge the Research FoundationFlanders (FWO) for individual support and project funding.

M. C. Soriano, and I. Fischer are with the Instituto de Física Interdisciplinar y Sistemas Complejos (IFISC), Consejo Superior Investigaciones Cientificas and Universitat de les Illes Balears, Campus Universitat Illes Balears, E-07122 Palma de Mallorca, Spain.

T. Berkvens, G. Van der Sande, G. Verschaffelt, and J. Danckaert are with the Department of Applied Physics and Photonics, Vrije Universiteit Brussel, Pleinlaan 2, 1050 Brussels, Belgium.

T. Berkvens, and I. Fischer have also been with the School of Engineering and Physical Sciences, Heriot-Watt University, Edinburgh EH14 4AS, Scotland.

J. Danckaert is also with the Department of Physics, Vrije Universiteit Brussel, Pleinlaan 2, 1050 Brussels, Belgium

Corresponding author: miguel@ifisc.uib-csic.es in the emission of semiconductor lasers can be compared to noisy fluctuations, in the sense that the laser produces high-dimensional, almost noise-like, dynamics over significant parameter ranges. In particular, we focus on the combined effects of the two external perturbations, extra current noise and optical feedback, on a single-mode semiconductor laser in order to explore how their described effects combine and interact. We perform our experiments on a Vertical-Cavity Surface-Emitting Laser (VCSEL), which allows single longitudinal and single transverse mode emission [9]. As most types of semiconductor lasers, the dynamics of VCSELs is affected by the presence of external optical feedback [10]-[12].

The past decade has witnessed a growing interest in the study of the effect of noise on semiconductor lasers subject to optical feedback. It has been shown that when a nonlinear semiconductor laser is subject to noise, new phenomena can arise. An example of such noise-induced phenomena is coherence resonance. Buldú and coworkers have analyzed the effect of the correlation of the external noise in an optical feedback system, looking for an optimal optical coherence resonance response in semiconductor lasers [13]. More recently, M. A. Arteaga et al. [14] used external current noise to study coherence resonance effects in a time-delayed bistable VCSEL, as isotropic optical feedback can induce polarization modehopping in VCSELs [15].

From the point of view of laser dynamics, it has also been shown that current noise can modify the dynamical regimes present in semiconductor lasers subject to feedback. T. Heil et al. studied stability properties and changes to the dynamics due to added noise to the injection current of a single-mode semiconductor laser subject to delayed optical feedback [16]. They concentrated on a feedback regime where there is coexistence of complex dynamics and stable emission on the external cavity mode with the highest gain (HGM). In order to investigate the stability properties of the HGM, current noise is added to the pump of the laser as a control parameter, demonstrating that the noise-induced escape from the HGM shows a distribution with exponential decay. Later, M. Yousefi found that realistic levels of carrier noise can influence the dynamics of a semiconductor laser subject to optical feedback [17]. Their results indicate that regular attractors, such as limit cycles or tori, tend to be more sensitive to carrier noise than to field noise. The stable emission states (fixed points) remain almost unaffected, apart from some broadening, and the same holds for the chaotic attractors. Following the line of these works, we will use the strength of the current noise as a control parameter to alter the dynamics of the laser with coherent feedback. While Refs. [16] and [17] deal with the effect of 
noise on the stability of different co-existing attractors, the system we study is always exhibiting complex dynamics with a single chaotic global attractor.

In the following sections, we will illustrate and analyze the interplay of extra current noise and chaotic emission dynamics of a semiconductor laser subject to delayed optical feedback. We present the first thorough experimental characterization of the influence of current noise in a wide range of feedback regimes. We finally argue that such systems with delay and noise are of high interest from a fundamental point of view, since they play an important role not only in laser systems, but also, for instance, in brain science and biology [18], [19].

\section{EXPERIMENTAL SETUP}

We have employed an oxide-confined vertical-cavity surface-emitting laser (VCSEL) lasing at $970 \mathrm{~nm}$, grown by the Optoelectronics Department of the University of Ulm [20]. The threshold current of the solitary laser is $I_{t h}=0.91 \mathrm{~mA}$ at $25{ }^{\circ} \mathrm{C}$. This device emits linearly polarized light in the fundamental mode until $I_{s w}=1.42 \mathrm{~mA}$, where it switches to the other orthogonal polarization mode. The switch occurs from the high-frequency mode to the low-frequency mode for increasing injection current.

The external optical feedback for the present study comes via the reflection from a distant gold mirror, in a long cavity configuration [21]. In the long cavity configuration, delay times of the feedback arm are longer than the relaxation oscillations (RO) period. In our experiments, the length of the external cavity is about $L_{e x t}=65 \mathrm{~cm}$, i.e. the delay time is $\tau=\frac{2 L_{e x t}}{c}=4.3 \mathrm{~ns}$. The RO period is $T_{R O}=0.4 \mathrm{~ns}$ when the VCSEL is biased at $I=1.2 \mathrm{~mA}$, being significantly shorter than the delay time. A schematic view of the experimental set-up is sketched in Figure 1. Therein, the feedback arm is enclosed by a gray dotted box. The feedback arm includes a beam-splitter, its reflection is used for the detection branch and its transmission for the feedback. In addition, the feedback arm includes a polarizer, to fix the linear polarization of the VCSEL and avoid polarization switching dynamics [22], a variable attenuator, which is a variable neutral density filter, and a gold mirror mounted on an adjustable gimbal mount.

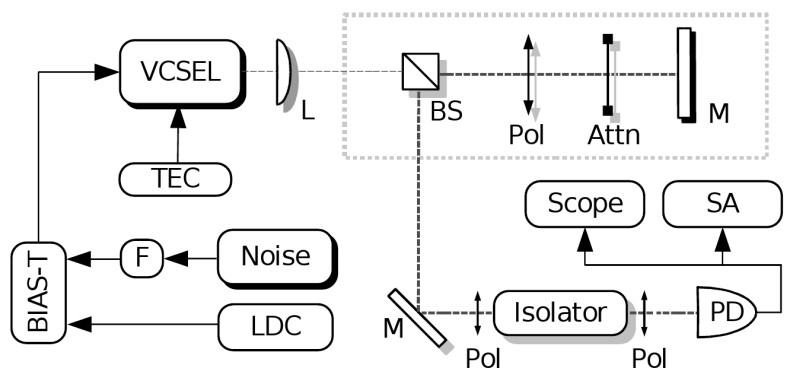

Fig. 1. Sketch of the experimental set-up. VCSEL: Vertical Cavity Surface Emitting Laser; Noise: noise generator; TEC: temperature controller; F: highpass filter; L: collimating lens; BS: beam-splitter; POL: polarizer; M: mirror; Attn: variable attenuator; PD: photo-diode; Isolator: optical isolator; LDC: laser driver controller; SA: spectrum analyzer

Prior to detection, the light emitted by the VCSEL passes through an optical isolator and is coupled into a single- mode fiber. We checked that the light emitted by the laser was linearly polarized along a fixed axis and no polarization switching dynamics occurred. As in [22], [23], the polarization of the emission is determined by the orientation of the polarizer in the optical feedback arm. Linearly polarized feedback ensures single-polarization mode emission if the orientation of the feedback axis matches the one of the laser emission. In contrast, VCSELs subject to isotropic feedback can operate in the two orthogonal polarization modes [24], [25].

The current noise is produced by an arbitrary waveform generator with a built-in independent real-time Gaussian noise generator (Tektronix AWG520). The current noise is restricted to low frequencies and it has a finite bandwidth. The AWG has a $3 \mathrm{~dB}$ bandwidth of $400 \mathrm{MHz}$. A high-pass filter, with a cutoff frequency of $1 \mathrm{MHz}$, has been placed at the output of the noise generator so that thermal effects due to the added current noise can be neglected. The thermal cut-off frequency of this laser has been measured to be around $200 \mathrm{KHz}$. A modulation with a frequency lower than the thermal cut-off could produce additional wavelength shifts due to thermal effects that shall be excluded from our investigation here. The current noise is then added to the driving low-noise pump source (Thorlabs LDC8002) by means of a bias-T and sent to the VCSEL.

\section{EXPERIMENTAL RESULTS AND DISCUSSION}

With the feedback configuration, as depicted in Fig. 1, we achieve a maximum threshold reduction of $5.6 \%$ due to the effect of the polarized optical feedback. This threshold reduction indicates that we work in a moderate feedback regime. Thanks to the variable attenuator in the feedback arm, we can tune the strength of the feedback. By tuning the feedback strength and the injection current, we have observed the typical feedback regimes including fully developed coherence collapse (CC) and low frequency fluctuations (LFFs). These feedback induced instabilities have been widely described in the literature [1], [7], [26]-[28].

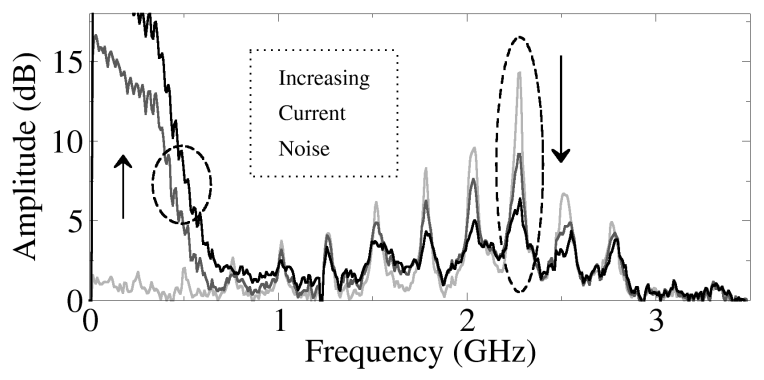

Fig. 2. Effects of the current noise in the RF spectra of a single-mode VCSEL subject to polarized optical feedback. Intermediate feedback strength. Bias current: $I=1.2 \mathrm{~mA}$. The strength of the current noise increases from the light gray curve to the dark gray and black curves. The plotted amplitude is the signal above the noise floor of the measurement apparatus.

In Figure 2, we show the influence of the current noise on the laser dynamics via the radio frequency (RF) spectra of the VCSEL emission. The operating conditions have been chosen such that the VCSEL is biased at $I=1.2 \mathrm{~mA}$, the strength of the polarized feedback is reduced $10 \mathrm{~dB}$ from the maximum 
feedback strength in our setup, and the solitary relaxation oscillation frequency is $f_{R O}=2.5 \mathrm{GHz}$ at this pump level. Figure 2 contains three RF spectra, corresponding to three different strengths of the current noise. The light gray, dark gray and black curves depict the RF spectra for the VCSEL with the addition of current noise of zero, intermediate and maximum strength, respectively. The maximum current noise strength corresponds to a root mean square (rms) value of $I_{r m s}=0.11 \mathrm{~mA}$, while the intermediate noise strength corresponds to $I_{r m s}=0.07 \mathrm{~mA}$. The peaks that appear around the solitary relaxation oscillation frequency $f_{R O}$ are spaced by $\Delta f=\frac{c}{2 L_{e x t}}=229 \mathrm{MHz}$, corresponding to the frequency difference of external cavity modes. Thus, the laser emission shows complex dynamical behavior on different external cavity modes. Because of the long delay, $\tau=4.3 \mathrm{~ns}$, the optical feedback generates a large number of external cavity modes, which appear by pairs through saddle-node bifurcations when the feedback strength is increased [1], [27], [29], [31].

The arrows in Fig. 2 show the trends of the main features in the RF spectrum when the current noise is increased. First, the average spectral power density below $f=400 \mathrm{MHz}$ increases due to the addition of the current noise. Second, the amplitude of the feedback peaks around $f=2 \mathrm{GHz}$ significantly diminish. The strong suppression of the feedback peaks with increasing current noise shows that the current noise has a damping effect on the delayed feedback-induced dynamics at this particular level of the feedback strength. The large amplitude and relatively narrow width of several frequency peaks in the shown RF spectrum illustrate the presence of dominating frequencies in the VCSEL emission, indicating certain periodicities in the dynamics or an underlying coherent chaotic attractor. The addition of current noise reduces the amplitude of those frequency peaks, i.e. the coherent oscillations in the VCSEL emission due to the optical feedback are disturbed when the current noise strength is increased.

Figure 3 provides a full overview of the experimental results at a bias current of $I=1.2 \mathrm{~mA}$. Firstly, the RF spectra in gray show the influence of the optical feedback without the inclusion of current noise. Secondly, the RF spectra in black show the influence of the optical feedback with added current noise $\left(I_{r m s}=0.1 \mathrm{~mA}\right)$. Finally, the feedback strength increases from Fig. 3.(a) to Fig. 3.(f).

Figure 3.(f) is obtained for the maximum strength of the feedback, which causes a laser threshold reduction of $5.6 \%$. A variable attenuator is placed in front of the gold mirror to gradually reduce the feedback strength. Figures 3.(a)-(e) are obtained for attenuations of $15 \mathrm{~dB}, 12 \mathrm{~dB}, 9.5 \mathrm{~dB}, 5.5 \mathrm{~dB}$ and $4.4 \mathrm{~dB}$, respectively.

It is apparent from Fig. 3 that the influence of the current noise on the feedback peaks is less relevant when the feedback level is increased. The current noise has a strong damping effect on the feedback-induced dynamics for low feedback strengths, as shown in Figs. 3.(a)-3.(c). The damping effect is less strong but still visible for intermediate feedback strengths, as can be seen in Figs. 3.(d)-3.(e). Interestingly, the damping of the feedback peaks has disappeared in Fig. 3.(f). Note that the feedback peaks in the absence of noise in Fig. 3.(f) are

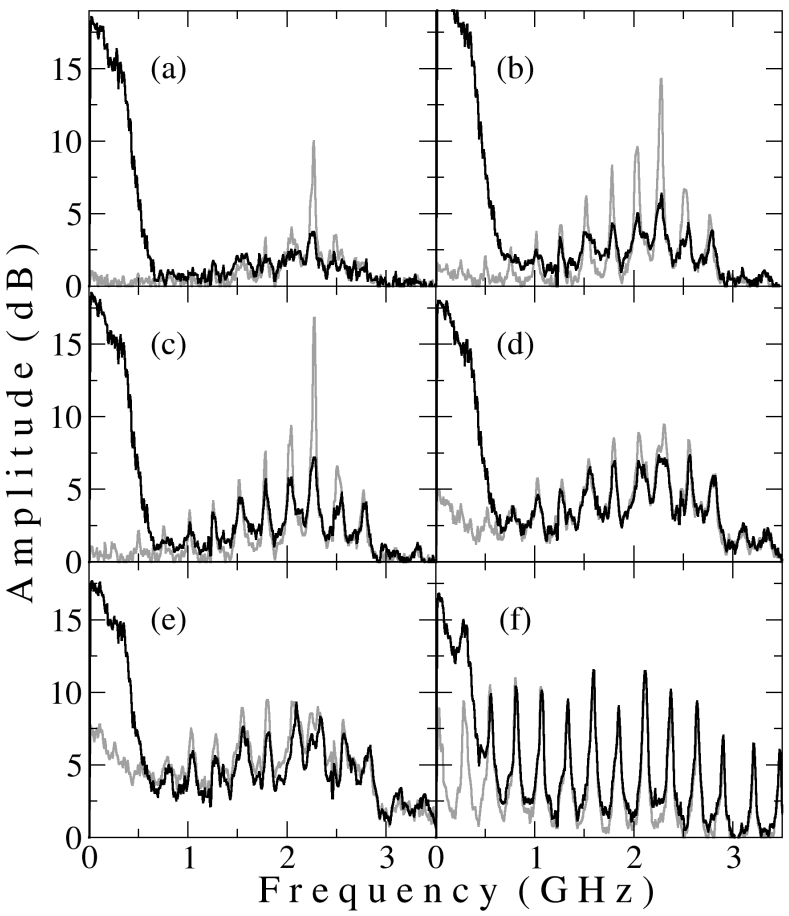

Fig. 3. Influence of the current noise in the RF spectra of a single-mode VCSEL subject to polarized optical feedback. The feedback strength increases from (a) to (f). Gray curves are obtained without current noise and black curves are obtained with a strong current noise. Bias current: $I=1.2 \mathrm{~mA}$.

present all over the detected RF spectrum, which ranges from 0 to $3.5 \mathrm{GHz}$ (limited by the bandwidth of the detector). This indicates that the laser exhibits complex dynamics at this particular feedback level [32], [33]. After the inclusion of current noise, we can see that the noise signature between 1 and $400 \mathrm{MHz}$ in the RF spectrum has been modified compared to the ones in Figs. 3.(a)-3.(e) as depicted in Fig. 3.(f), see black line. Particularly, we see that the feedback peak around $300 \mathrm{MHz}$ emerges from the current noise background.

The temporal instabilities of the laser emission associated with the experimental results presented in Figs. 3.(a)-3.(f) can be inferred from the shown RF spectra. On the one hand, the optical feedback excites relaxation oscillation dynamics for low feedback strengths, see Figs. 3.(a)-3.(c). On the other hand, the optical feedback induces Low Frequency Fluctuations (LFFs) dynamics for larger feedback strengths, see the increase of the amplitude of the measured RF spectra in the absence of noise at low frequencies (below $500 \mathrm{MHz}$ ), typical of LFF dynamics.

Similar results are obtained for higher injection currents. Fig. 4 shows the RF spectra measured at a bias current of $I=1.4 \mathrm{~mA}$. Again, the current noise increases the damping of the feedback peaks around $3 \mathrm{GHz}$ for an intermediate feedback level corresponding to a feedback attenuation of $9 \mathrm{~dB}$, see Fig. 4.(a), while it has no influence on the feedback peaks for a larger feedback strength corresponding to a feedback attenuation of $1.5 \mathrm{~dB}$, as can be seen in Fig. 4.(b). The influence of the current noise appears to be frequency dependent in Fig. 4.(a), i.e. the amplitude of the feedback peaks around 
$3 \mathrm{GHz}$ diminishes with the addition of extra current noise, while the peaks around $2 \mathrm{GHz}$ are unmodified. This shows that the current noise has a stronger influence on the peaks that appear around $f_{R O}$, which increases when the injection current is increased.

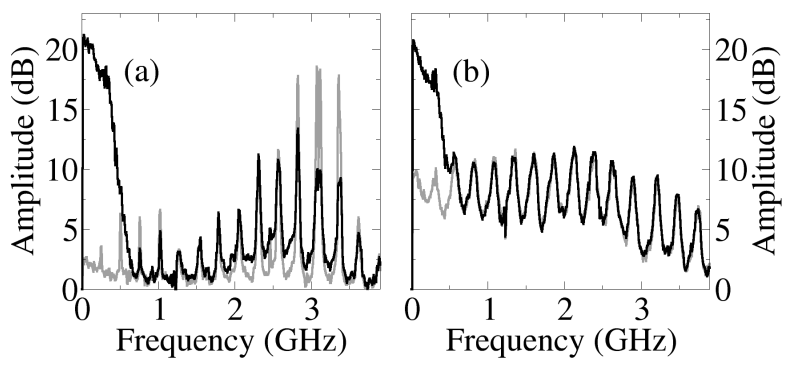

Fig. 4. Influence of the current noise in the RF spectra of a single-mode VCSEL subject to polarized optical feedback. (a) Moderate feedback strength. (b) Maximum feedback strength. Gray curves are obtained without current noise and black curves are obtained with a strong current noise. Bias current: $I=1.4 \mathrm{~mA}$.

The results presented in [8] hint at an increase of the nonlinear saturation when the current noise is increased. However, the lowering of the feedback peaks in Figs. 3.(a)-(e) and Fig. 4.(a) seems not to be only related to an increase of the nonlinear saturation when the current noise is increased. An increase of the nonlinear saturation could indeed explain a reduction in the amplitude of the feedback peaks, but it would be accompanied by a similar lowering in the amplitude levels of the valleys between these feedback peaks, which rather increase or are unaffected in the experiments. Therefore, another mechanism must be dominating in our case. We discuss the origin of the reported damping in the amplitude of the RF spectrum frequency peaks in the section devoted to modelling and numerical results.

Finally, Fig. 3.(f) and Fig. 4.(b) show that the damping influence of the current noise is no longer present when the emission of the laser shows fully developed dynamics, i.e. when there are feedback peaks all over the detected RF spectrum. In the following section, we perform numerical simulations of a Lang-Kobayashi rate-equations model [29] in order to gain information on the origin of the observed behavior.

\section{DESCRIPTION OF THE MODEL}

A single-transverse and single-polarization mode VCSEL subject to polarized optical feedback can be modeled via rate equations for the slowly varying amplitude of the electric field $E(t)$ and the carrier number $N(t)$. The use of polarized optical feedback ensures single-polarization mode emission if the orientation of the polarizer in the feedback arm matches the one of the laser emission [22]. We have checked that the addition of current noise in the presence of polarized optical feedback does not induce polarization dynamics in our device. Hence, we focus on the LK model being, despite its simplifications, a good model to reproduce our experimental findings. The equations for the dynamical evolution of $E(t)$ and $N(t)$ are given by [29]:

$$
\begin{gathered}
\dot{E}(t)=\frac{1+i \alpha}{2}\left[G(t)-\gamma_{p}\right] E(t)+\kappa E(t-\tau) e^{-i \Phi}+F_{E}(t), \\
\dot{N}(t)=\frac{I+I_{C}(t)}{e}-\frac{N(t)}{\tau_{N}}-G(t)|E|^{2}+F_{N}(t),
\end{gathered}
$$

where the gain is defined as $G=g\left(N / V-n_{o}\right) /\left(1+\epsilon|E|^{2}\right)$. $I_{C}(t)$ represents the low frequency current noise added in the experiments and corresponds to a Gaussian white noise passed through a fifth order Butterworth filter. The Butterworth filter is designed to have its cutoff frequency at $\omega=2 \pi \times 400 \mathrm{MHz}$. The line width enhancement factor has been chosen as $\alpha=3.5$, the differential gain is $g=$ $2.12 \times 10^{-4} \mathrm{~ns}^{-1}$, the active region volume is $V=1.6 \mu \mathrm{m}^{3}$, the carrier density at transparency is $n_{o}=4 \times 10^{6} \mu \mathrm{m}^{-3}$, the gain compression factor is $\epsilon=3.375 \times 10^{-7}$, the photon decay rate is $\gamma_{p}=0.5 \mathrm{ps}^{-1}, q$ is the elementary charge, the electron-hole recombination life-time is $\tau_{N}=1.8 \mathrm{~ns}$. These parameter values have been adjusted to reproduce the experimental solitary laser threshold current of $I_{t h}=0.91 \mathrm{~mA}$ and the RO frequency at $I=1.2 \mathrm{~mA}$ of $f_{R O}=2.5 \mathrm{GHz}$. The feedback strength $\kappa$ will be varied in the numerical simulations, the external cavity delay time is $\tau=4.3 \mathrm{~ns}$, and a constant feedback phase is taken of $\phi=0$. The Langevin noise sources $F_{E}(t)$ and $F_{N}(t)$ account for the intrinsic noise, and satisfy $\left\langle F_{i}(t) F_{i}\left(t^{\prime}\right)=2 D_{i i} \delta\left(t-t^{\prime}\right)\right\rangle$, with $D_{E E}=R_{s p}$ and $D_{N N}=N / \tau_{N}$, where $R_{s p}=1 \times 10^{10} \mathrm{~s}^{-1}$ is the rate of spontaneous emission into the lasing mode above lasing threshold.

As demonstrated in [17], both field and carrier noise need to be included in the simulations to properly model semiconductor laser dynamics. It is known that field noise contributes to the line-width of the laser in continuous wave $(\mathrm{CW})$ operation [30]. While carrier noise does not influence the line-width of the laser, it can modify the stability of the attractors when the laser exhibits complex dynamics [17].

In Figure 5, we show the RF spectra of the numerical results with and without the addition of current noise for increasing feedback strength. The feedback strength values can be read in the figure, ranging from $\kappa=0.5 \mathrm{~ns}^{-1}$ to $\kappa=20 \mathrm{~ns}^{-1}$; while the values of the current noise are $I_{r m s}=0$ (no noise) and $I_{r m s}=0.09 \mathrm{~mA}$ (maximum noise).

The numerical results are in good qualitative agreement with the experimental results. The current noise modifies the feedback dynamics at low feedback strength values, while it has no significant influence on the dynamics at large feedback strength values. In addition, the numerical simulations provide additional information on the influence of the current noise on the dynamics since we have access to the phase of the electric field. The electric field can be written as $E=\sqrt{P} \exp [j \phi(t)]$, where $\phi(t)$ is the phase of the electric field. In semiconductor laser feedback dynamics, it is useful to introduce the delay phase difference as $\Delta \phi(t)=\phi(t)-\phi(t-\tau)$, since this quantity contains information about how the laser reacts to the coherent feedback and how it visits the different external cavity modes [27]. A constant value of the phase difference $\Delta \phi(t)$ means that the laser is emitting in a single cavity mode, 


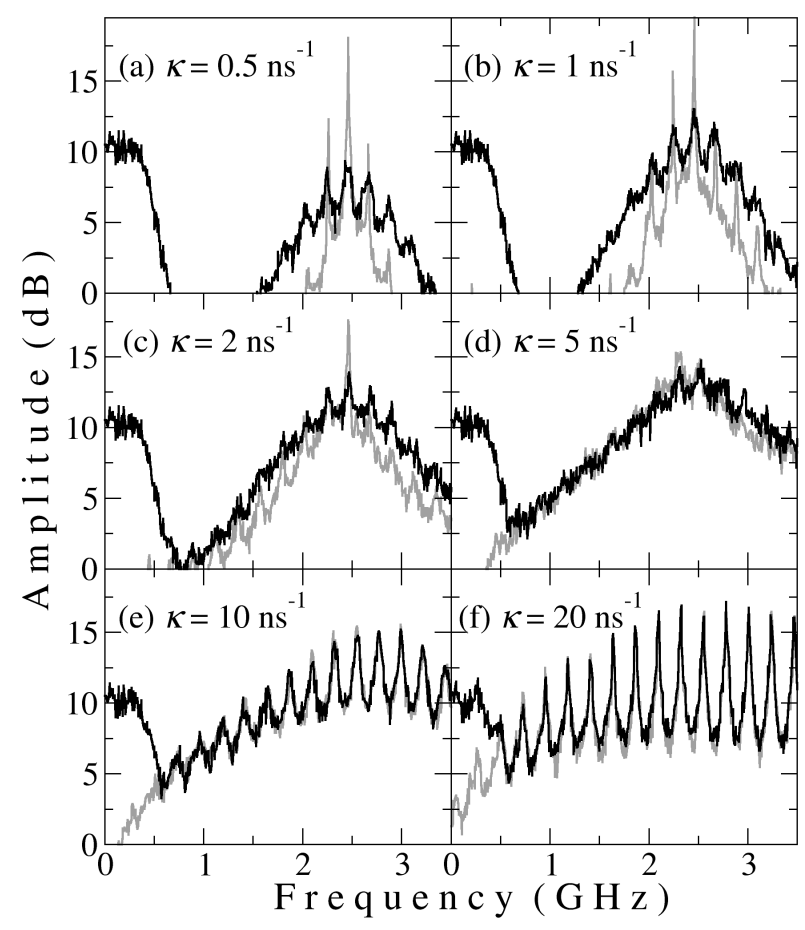

Fig. 5. Numerical results on the influence of the current noise on the RF spectra of a single-mode VCSEL subject to polarized optical feedback. The feedback strength increases from (a) to (f). Gray curves are obtained without current noise and black curves are obtained with a strong current noise. Bias current: $I=1.2 \mathrm{~mA}$. The plotted amplitude has been shifted by an arbitrary amount.

while phase-slips of $2 \pi$ correspond to jumps among different external cavity modes or their attractor ruins. Therefore, from the dynamics of $\Delta \phi(t)$ a lot of information can be gained about the modal and phase dynamics related to the feedback instabilities.

We have checked the temporal evolution of the phase difference $\Delta \phi(t)$ in the numerical simulations with and without noise. Figure 6 illustrates $\Delta \phi(t)$ for low values of the feedback strength $\left(\kappa<2 \mathrm{~ns}^{-1}\right)$, showing that there exist phase difference jumps between two distinct levels $(\Delta \phi(t)=0$ and $\Delta \phi(t) \approx-6)$. The two distinct levels correspond to different external cavity modes. These external cavity modes are not fixed points of the dynamics but ghosts (attractor ruins) of local attractors which have merged to a global chaotic attractor. When the system is in the vicinity of one of the external cavity modes, the phase space trajectory exhibits coherent oscillations around the external cavity modes. Please note that coherent oscillations refers in this context not to optical coherence, but to the concept of nonlinear dynamics that oscillations around a single center in phase space are called coherent. When the current noise is present, the phase difference jumps between the ruins of external cavity modes become more frequent in time, as illustrated in Figure 6(b).

If we define $\Delta t$ as the mean time spent on one attractor ruin of an external cavity mode, we can quantify the influence of the current noise on the delayed-feedback dynamics as a function of the feedback strength by using a threshold procedure. The values of $\Delta t$ as a function of the feedback

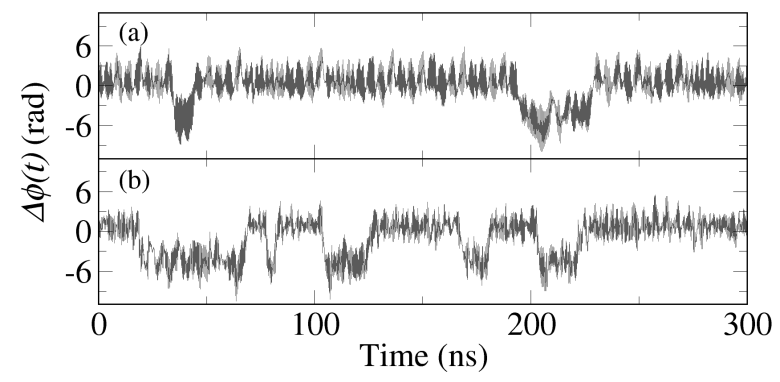

Fig. 6. Numerical results on the influence of the current noise strength on the phase difference of a single-mode VCSEL subject to polarized optical feedback $\left(\kappa=1 \mathrm{~ns}^{-1}\right)$. (a) $I_{r m s}=0$ and (b) $I_{r m s}=0.09 \mathrm{~mA}$. Bias current: $I=1.2 \mathrm{~mA}$.

strength, with and without current noise, are shown in Fig. 7. For $\kappa=1 \mathrm{~ns}^{-1}$, we find $\Delta t=66.5 \mathrm{~ns}$ in the absence of current noise $\left(I_{r m s}=0\right)$ and $\Delta t=26.7 \mathrm{~ns}$ in the presence of current noise $\left(I_{r m s}=0.09 \mathrm{~mA}\right)$. For $\kappa=2 \mathrm{~ns}^{-1}$, we find that $\Delta t=8.7 \mathrm{~ns}$ when $I_{r m s}=0$ and $\Delta t=7.3 \mathrm{~ns}$ when $I_{r m s}=0.09 \mathrm{~mA}$. Since $\Delta t$ exhibits a certain statistical distribution, all given values are an average over time. The lower the values of $\Delta t$, when the current noise is increased, indicate that the laser dynamics is more unstable and the coherent periodicities are disturbed by the external cavity mode hopping. When the feedback strength is larger $\left(\kappa>5 \mathrm{~ns}^{-1}\right)$ the temporal evolution of the phase difference shows a very fast chaotic itinerancy among the external cavity modes even in the absence of current noise. The corresponding phase difference jumps occur so often that no coherent oscillations around one mode can build up. Consequently, the influence of noise reduces significantly and we do not find changes in the RF spectra when the noise strength is increased, as shown in Fig. 7. Therefore, as long as the feedback instabilities comprise coherent oscillations, related to oscillations around individual external cavity modes, noise can have a strong effect on the dynamics and on the statistics of the mode jumps. As soon as the deterministic dynamics already exhibits frequent mode jumps, and no coherent oscillations can build up, the additional influence of noise can not be easily identified anymore.

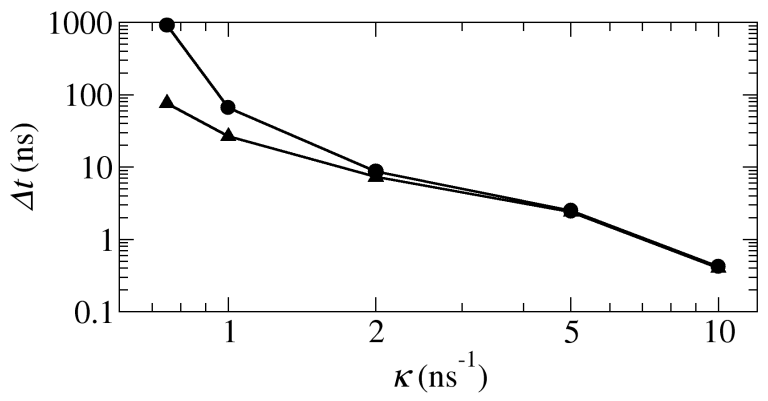

Fig. 7. Numerical results on the influence of the current noise strength on the time between phase difference jumps $\Delta t$ of a single-mode VCSEL subject to polarized optical feedback as a function of the feedback strength $\kappa$. Circles for $I_{r m s}=0$ and triangles for $I_{r m s}=0.09 \mathrm{~mA}$. Bias current: $I=1.2 \mathrm{~mA}$. 


\section{Conclusions And OUTlooK}

In conclusion, the influence of the current noise in a semiconductor laser subject to polarized optical feedback is twofold. On the one hand, the current noise induces a damping of the feedback peaks for weak and intermediate feedback levels, i.e. before the system enters the regime with fully developed coherence collapse dynamics. On the other hand, the effect of the current noise is not recognizable in the RF spectra when the response of the laser is in a state of fully developed coherence collapse. This illustrates the rich phenomenology that appears when delayed feedback and strong noise fluctuations interact.

According to the experimental and numerical results, the extra current noise has a larger influence on the laser dynamics whose attractors still exhibit some local regularity (local coherent oscillations) than on fully developed chaotic attractors. In the maximum feedback regime, the feedback peaks are spread all over the detected RF spectrum, which ranges from 0 to $3.5 \mathrm{GHz}$. In this regime, the influence of the low frequency current noise in the laser dynamics is minimal. In contrast, for lower feedback strengths, the feedback peaks do not appear at frequencies lower than $400 \mathrm{MHz}$ and the extra current noise modifies the feedback induced dynamics.

In order to explain the interplay of noise and delayed feedback dynamics, we have analyzed via numerical simulations how the current noise modifies the laser dynamics, from low to moderate optical feedback levels. The observed changes in the RF spectra are reflected in the different way in which the external cavity modes are visited when the current noise is added, i.e. coherent oscillations around a single external cavity mode are disturbed in the presence of extra current noise.

In conclusion, extra current noise and delayed optical feedback can modify the dynamical properties of single-mode semiconductor lasers, which are nonlinear optical oscillators. We have illustrated that, when both perturbations are applied simultaneously to the laser, the modification of the dynamical properties is not only a linear superposition of the individual effects but there can be a delicate interplay between the two, depending on the operation conditions.

\section{ACKNOWLEDGMENTS}

MCS thanks the MICINN for a "Juan de la Cierva" contract. GVDS is a Postdoctoral Fellow of the FWO.

\section{REFERENCES}

[1] J. Mørk, B. Tromborg, and J. Mark, "Chaos in semiconductor lasers with optical feedback: theory and experiment", IEEE J. Quantum Electron. vol. 28, pp. 93-108, 1992.

[2] D. H. DeTienne, G. R. Gray, G. P. Agrawal, and D. Lenstra, "Semiconductor Laser Dynamics for Feedback from a Finite-Penetration-Depth Phase-Conjugate Mirror", IEEE J. Quantum Electron., vol. 33, pp. 838844, 1997.

[3] J. P. Goedgebuer, L. Larger, and H. Porte, "Optical Cryptosystem Based on Synchronization of Hyperchaos Generated by a Delayed Feedback Tunable Laser Diode”, Phys. Rev. Lett., vol. 80, pp. 2249-2252, 1998.

[4] S. Wieczorek, B. Krauskopf, and D. Lenstra, "A unifying view of bifurcations in a semiconductor laser subject to optical injection", Opt. Comm., vol. 172, pp. 279-295, 1999.

[5] G. Van der Sande, M. C. Soriano, I. Fischer, and C. R. Mirasso, "Dynamics, correlation scaling, and synchronization behavior in rings of delay-coupled oscillators", Phys. Rev. E, vol. 77, 055202(R), 2008.
[6] J. Sacher, D. Baums, P. Panknin, W. Elsässer, and E.O. Göbel, "Intensity instabilities of semiconductor lasers under current modulation, external light injection, and delayed feedback", Phys. Rev. A, vol. 45, pp. 1893 1905, 1992.

[7] B. Krauskopf and D. Lenstra, "Fundamental issues of nonlinear laser dynamics", Dynamics of semiconductor Lasers workshop, Texel, The Netherlands, AIP Conference Proceedings 548, 2000, American Institute of Physics.

[8] G. Van der Sande, M. C. Soriano, M. Yousefi, M. Peeters, J. Danckaert, G. Verschaffelt, and D. Lenstra, "Influence of current noise on the relaxation oscillation dynamics of semiconductor lasers", Appl. Phys. Lett. vol. 88, 071107, 2006.

[9] H. E. Li, K. Iga, Vertical-cavity surface-emitting laser devices (Springer, 2002).

[10] Y. C. Chung and Y. H. Lee, "Spectral characteristics of vertical-cavity surface-emitting laser with optical feedback", IEEE Photon. Technol. Lett., vol. 3, pp. 597-599, 1991.

[11] S. Jiang, Z. Pan, M. Dagenais, R. A. Morgan, and K. Kojima, "Influence of external optical feedback on threshold and spectral characteristics of vertical-cavity surface-emitting lasers", IEEE Photon. Technol. Lett., vol. 6, pp. 34-36, 1994

[12] P. Besnard, F. Robert, M. L. Chares, and G. Stephan, "Theoretical modeling of vertical-cavity surface-emitting lasers with polarized optical feedback", Phys. Rev. A, vol. 56, pp. 3191-3205, 1997.

[13] J. M. Buldú, J. García-Ojalvo, C. R. Mirasso, M. C. Torrent, and J. M. Sancho, "Effect of external noise correlation in optical coherence resonance", Phys. Rev. E, vol. 64, 051109, 2001.

[14] M. A. Arteaga, M. Valencia, M. Sciamanna, H. Thienpont, M. LópezAmo, and K. Panajotov, "Experimental evidence of coherence resonance in a time-delayed bistable system”, Phys. Rev. Lett. vol. 99, 023903, 2007.

[15] M. Sciamanna, K. Panajotov, H. Thienpont, I. Veretennicoff, P. Mégret, and M. Blondel, "Optical feedback induces polarization mode hopping in vertical-cavity surface-emitting lasers”, Opt. Lett. vol. 28, pp. 1543-1545, 2003.

[16] T. Heil, I. Fischer, and W. Elsässer, "Stabilization of feedback-induced instabilities in semiconductor lasers", J. Opt. B: Quantum Semiclass. Opt., vol. 2, no. 3, pp. 413-420, 2000.

[17] M. Yousefi, D. Lenstra, and G. Vemuri, "Carrier inversion noise has important influence on the dynamics of semiconductor lasers", IEEE J. Sel. Top. Quantum Electron., vol. 10, pp. 955-960, 2004.

[18] G. Decoa, V. Jirsac, A. R. McIntoshe, O. Spornsf, and R. Kötterg, "Key role of coupling, delay, and noise in resting brain fluctuations", Proc. Natl. Acad. Sci., vol. 106, pp. 10302-10307, 2009.

[19] D. Bratsun, D. Volfson, L. S. Tsimring, and J. Hasty, "Delay-induced stochastic oscillations in gene regulation", Proc. Natl. Acad. Sci., vol. 102 pp. 14593-14598, 2005.

[20] H. Unold, S. Mahmoud, R. Jaeger, M. Grabherr, R. Michalzik, and K. Ebeling, "Large-area single-mode vcsels and the self-aligned surface relief”, IEEE J. Sel. Top. Quantum Electron., vol. 7, pp. 386-392, 2001.

[21] T. Heil, I. Fischer, W. Elsässer, and A. Gavrielides, "Dynamics of Semiconductor Lasers Subject to Delayed Optical Feedback: The Short Cavity Regime", Phys. Rev. Lett. vol. 87, no. 24, pp. 243901, 2001.

[22] M. C. Soriano, M. Yousefi, J. Danckaert, S. Barland, M. Romanelli, G. Giacomelli, and F. Marin, "Low-frequency fluctuations in vertical-cavity surface-emitting lasers with polarization selective feedback: Experiment and theory”, IEEE J. Sel. Top. Quantum Electronics, vol. 10, no. 5, pp. 998-1005, 2004

[23] G. Giacomelli, F. Marin, and M. Romanelli, "Multi-time-scale dynamics of a laser with polarized optical feedback", Phys. Rev. A, vol. 67, pp. $053809,2003$.

[24] C. Masoller and N. B. Abraham, "Low-frequency fluctuations in verticalcavity surface-emitting semiconductor lasers with optical feedback", Phys. Rev. A, vol. 59, no. 4, pp. 3021-3031, 1999.

[25] M. Giudici, S. Balle, T. Ackemann, S. Barland, and J. R. Tredicce, "Polarization dynamics of vertical-cavity surface-emitting lasers with optical feedback: experiment and model", J. Opt. Soc. Am. B, vol. 16, pp. 2114-2123, 1999

[26] D. Lenstra, B. Verbeek, and A. Den Boef, "Coherence collapse in singlemode semiconductor lasers due to optical feedback", IEEE J. Quantum Electron. vol. 21, pp. 674-679, 1985.

[27] T. Sano, "Antimode dynamics and chaotic itinerancy in the coherence collapse of semiconductor lasers with optical feedback", Phys. Rev. A, vol. 50, no. 3, pp. 2719-2726, 1994.

[28] T. Heil, I. Fischer, W. Elsässer, "Coexistence of Low Frequency Fluctuations and stable emission on a single high-gain mode in semiconductor lasers with external optical feedback", Phys. Rev. A, vol. 58, no. 4, pp. 2672-2675(R), 1998. 
[29] R. Lang and K. Kobayashi, "External optical feedback effects on semiconductor injection laser properties", IEEE J. Quantum Electron. vol. 16 , pp. 347-355, 1980.

[30] C. H. Henry, "Theory of the linewidth of semiconductor lasers", IEEE J. Quantum Electron., vol. 18, pp. 259-264, 1982.

[31] T. Erneux, F. Rogister, A. Gavrielides, and V. Kovanis, "Bifurcation to mixed external cavity mode solutions for semiconductor lasers subject to optical feedback", Opt. Comm. vol. 183, nos. 5-6, pp. 467-477, 2000.

[32] J. Wang and K. Petermann, "Noise analysis of a semiconductor laser within the coherence collapse regime", IEEE J. Quantum Electron. vol. 27, pp. 3-9, 1991 .

[33] I. Fischer, G. H. M. van Tartwijk, A. M. Levine, W. Elsässer, E. Göbel, and D. Lenstra, "Fast pulsing and chaotic itinerancy with a drift in the coherence collapse of semiconductor lasers", Phys. Rev. Lett. vol. 76, pp. 220-223, 1996.

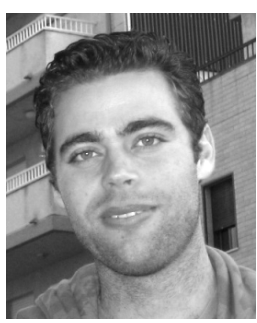

Miguel Cornelles Soriano was born in Benicarlo, Spain, in 1979. He received the Telecommunications Engineering degree from the Technical University of Catalonia, Spain, in 2002 and the Ph.D. degree in Engineering from the Free University of Brussels, Belgium, in 2006.

Since January 2008, he holds a "Juan de la Cierva" scientific contract at the Instituto de Física Interdisciplinar y Sistemas Complejos (IFISC), Palma de Mallorca, Spain. His main research interests include optical feedback, theoretical modeling and synchronization properties of semiconductor lasers.

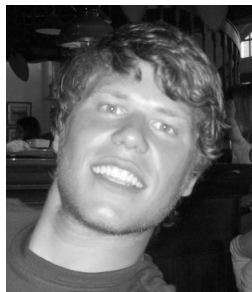

Thomas Berkvens was born in Antwerp, Belgium in 1982. He received his degree in Electrotechnica Engineering from the Vrije Universiteit Brussel, Belgium in 2005. He was a teaching and research assistant at this institute from 2005 to 2008. In 2007 he stayed at Heriot-Watt University, Edinburgh. During these years he perfomed research on the dynamics of semiconductor lasers subject to optical feedback. He recently joined the Belgian Nuclear Research Centre SCK-CEN (Mol, Belgium) as a scientific collaborator in Education and Training

projects.

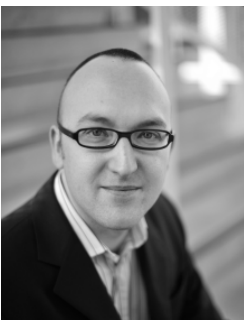

Guy Van der Sande (Mechelen, 1978) graduated in 2001 at the Vrije Universiteit Brussel (VUB, Belgium) as an electrotechnical engineer with majors in photonics. In 2005, he received the title of Doctor in the Applied Sciences at the department of Applied Physics and Photonics (TONA) of the VUB. His $\mathrm{PhD}$ was focused on theoretical modelling of vertical-cavity surface-emitting lasers and nonlinear photonic crystals. After his $\mathrm{PhD}$, he stayed for one year at the department Optique Nonlinéaire Théorique of the Université Libre de Bruxelles. In 2006, he returned to TONA-VUB as a postdoctoral research fellow of the Flemish Fund for Scientific Research (FWO-Vlaanderen), where he is still today. In 2007, he was a visiting scientist at the Institute for CrossDisciplinary Physics and Complex Systems, IFISC (UIB-CSIC), Spain. His research interests are now focused on nonlinear dynamics of delay-coupled semiconductor lasers and of semiconductor ring lasers.

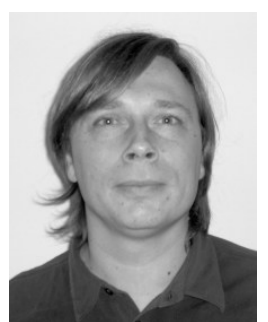

Guy Verschaffelt was born in Belgium in 1973. He received the M.E. degree in photonics and the Ph.D. degree from the Vrije Universiteit Brussel (VUB), Brussels, Belgium in 1996 and 2000, respectively. Presently, he is a postdoctoral researcher with VUB. His research interests include polarization and noise properties of vertical-cavity surface-emitting lasers, emission properties of broad-area high power semiconductor lasers and the dynamics of semiconductor ring lasers.

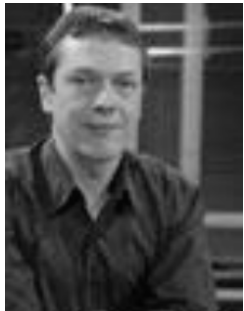

Jan Danckaert was born in Antwerp (Belgium) in 1964, and graduated in Physics at the University of Antwerp in 1985. He then joined the Department of Applied Physics and Photonics (TONA) of the Vrije Universiteit Brussel (VUB, Brussels, Belgium) as a teaching assistant. In 1992, he obtained a $\mathrm{PhD}$ on the subject of nonlinear optics, studying the response of optical resonators with a third order nonlinear susceptibility. After a stay abroad at INPG in Grenoble, France (1993), he returned to the same department of the VUB as a postdoctoral research fellow of the Research Foundation Flanders (FWO). In 2001 and 2002 he was a visiting scientist at IFISC, Palma de Mallorca, Spain. As from 20052006 on he is a full-time professor at the VUB, teaching introductory physics for both science and (bio-) engineering students, and courses in photonics at Master level. His current research interests go towards semiconductor laser dynamics, VCSELs and semiconductor ring lasers in particular, and nonlinear dynamics in general. As an author or co-author, he contributed to more than 50 papers in international refereed journals and 100 contributions to international conferences of which 5 invited talks. He is a member of IEEE, EPS and OSA. $\mathrm{He}$ is also active in advisory panels on science policy in Flanders, and regularly organises and/or performs in science popularisation initiatives.

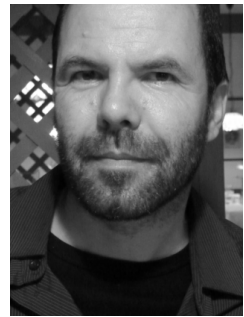

Ingo Fischer received his diploma and Ph.D. degrees in physics from Philipps University, Marburg, Germany, in 1992 and 1995, respectively. He stayed at TU Darmstadt, Germany, from 1995 to 2004 and at Vrije Universiteit Brussel, Belgium, from 2005 to 2007. In 2007 he became full professor in Photonics at Heriot-Watt University, Edinburgh, U.K. Since 2009 he is professor at the Institute for CrossDisciplinary Physics and Complex Systems, IFISC (UIB-CSIC). His studies are focused on nonlinear photonics and brain dynamics. In particular, he is concentrating on emission properties and dynamics of modern photonic sources, coupled laser systems, synchronization of lasers and neurons, and utilization of chaos. He received the Research Prize of the Adolf-Messer Foundation in 2000 and the first Hassian Cooperation Prize of the Technology Transfer Network in 2004. 